\title{
Geometric Brownian Motion Assumption and Generalized Hyperbolic Distribution on Modeling Returns
}

\author{
Ivivi J. Mwaniki \\ School of Mathematics, University of Nairobi, Kenya \\ Email: jimwaniki@uonbi.ac.ke
}

\begin{abstract}
Generalized hyperbolic distribution and some of its subclasses like normal, hyperbolic and variance gamma distributions are used to fit daily log returns of eight listed companies in Nairobi Securities Exchange and Montréal Exchange. EM-based maximum likelihood estimation procedure is used to estimate parameters of the model. Kernel densities and empirical distribution of data are compared. The goodness of fit statistics of proposed distributions are used to measure how well model fits the data. Empirical results show that Generalized hyperbolic Distribution seems to improve partially, the geometric Brownian assumption on modeling returns of the underlying process, both in a developed and emerging market. Both markets seem to have different stochastic time.
\end{abstract}

Keywords: Emerging market, Generalized hyperbolic distribution, Calibration, goodness of fit.

\section{Introduction}

Geometric Brownian motion assumption for modeling log returns is used in the Black and Scholes[1] and Merton[2] methodology. This has become the dominant paradigm for valuing options and other derivatives. The method uses a delta hedging argument to value options based on the absence of arbitrage strategies that profit instantaneously. Despite the success of the Black Scholes model assumption, there is a non-negligible discrepancy between the model and the real market data. We set out to investigate the geometric assumption of modeling the underlying asset in Kenya's Nairobi securities exchange and Canada Montréal exchange, as well as confirming some of the stylized facts documented by Cont[3].

To incorporate the asymmetric and leptokurtic features in asset pricing, Madan and Senata[4] and [5] studied a time changed Brownian Motion in the context of asymmetric variance gamma process. Among these models, (see Car and $\mathrm{Wu}[6]$,Eberlein and Keller[7] etc), several studies have applied the exponential generalized hyperbolic Lévy motion and its subclasses to study log returns. We focus our attention to the generalized hyperbolic(GH), Hyperbolic(HY) and Variance Gamma(VG) and normal(NM) distributions in modeling log returns. The Generalized Hyperbolic distribution was first introduced by Barndoff-Nielsen[8] in the context of the sand project. In 1995 Eberlein and Keller[7] introduced and applied the hyperbolic distribution, to price vanilla options based on German stocks.

In this paper we use empirical evidence to investigate the basic geometric Brownian assumption on modeling log returns. In addition, we compare kernel density estimates against the maximum likelihood estimate of generalized hyperbolic distribution and some of its subclasses like the hyperbolic and the variance gamma distributions of daily log returns of eight listed companies i.e. (four from each exchange) in Montréal and Nairobi Securities Exchange.

The rest of this paper is organized as follows. Section 2 presents data description and empirical evidence of imperfections log-normality assumption of modeling equities. In Section 3, the generalized hyperbolic distribution and some of its subclasses, hyperbolic and variance gamma are defined. Details of parameter estimation are presented. Section 4, Kolmogorov distance and frequency distribution are used to test the normality assumption and proposed model fit. Section 5 concludes.

\section{Normality Assumption on Log Returns}

In the Black-Scholes world, the financial asset is modeled by geometric Brownian motion. For equities, the model assumes that the price process $S=\left\{S_{t}, t \geq 0\right\}$ of an asset is governed by stochastic differential 
equation

$$
d S_{t}=\mu S_{t} d t+\sigma S_{t} d B_{t}, \Rightarrow S_{t}=S_{0} \exp \left(\left(\mu-\frac{\sigma^{2}}{2}\right) t+\sigma B_{t}\right)
$$

where $\mu$ is the drift, $\sigma$ is the assumed market constant volatility and $B_{t}$ is standard Brownian motion. This implies that

$$
\log _{e} S_{t} \sim N\left(\log _{e} S_{0}+\left(\mu-\frac{\sigma^{2}}{2}\right) t, \sigma^{2} t\right), \quad \forall t>0
$$

\subsection{Data Description and the Empirical Distribution}

A sample of eight listed companies were collected from Nairobi Securities Exchange(NSE), Barclay's Bank of Kenya (BBK), Kenya Commercial Bank (KCB), East Africa Breweries ltd (EABL), Kenya Airways (KQ and the restof the data was downloaded from yahoo finance for Montérial exchange(Mx), i.e) Angnico Eagle Mines limited (AEM), Alcan Inc (AL), Royal Bank of Canada (RY)and Sun life Financial (SLF). Daily adjusted closing prices from January 4th, 2000 to August 30th, 2005, were used to determine daily $\log$ returns. Let $S_{j}:=S\left(t_{j}\right)$ be the price on day $t_{j}, j=0,1,2, \ldots, n-1$. Sample increments of log returns is defined by $x_{j}=\log S_{j}-\log S_{j-1}, \quad j=1,2, \ldots, n-1$. We are guided by the geometric Brownian motion assumption that the sequence $\left(x_{j}\right)_{0<j<n-1}$ is independent and identically distributed to model stock prices $S=\left(S_{t}\right)_{t>0}$. The discrete financial time series data correspond to the value of the continuous time process $S$ at equidistant integer points. Therefore, to obtain a discrete time series from our continuous model, we shall consider

$$
S_{n}=S_{0} \exp \left(\sum_{j=1}^{n} x_{j}\right) .
$$

As an illustration in Figure 1 we use the kernel density versus maximum likelihood fit of the normal distribution of daily log returns for Kenya Commercial Bank (KCB) and Royal Bank of Canada (RY) daily log returns respectively.

Empirical Density vs Fitted Normal

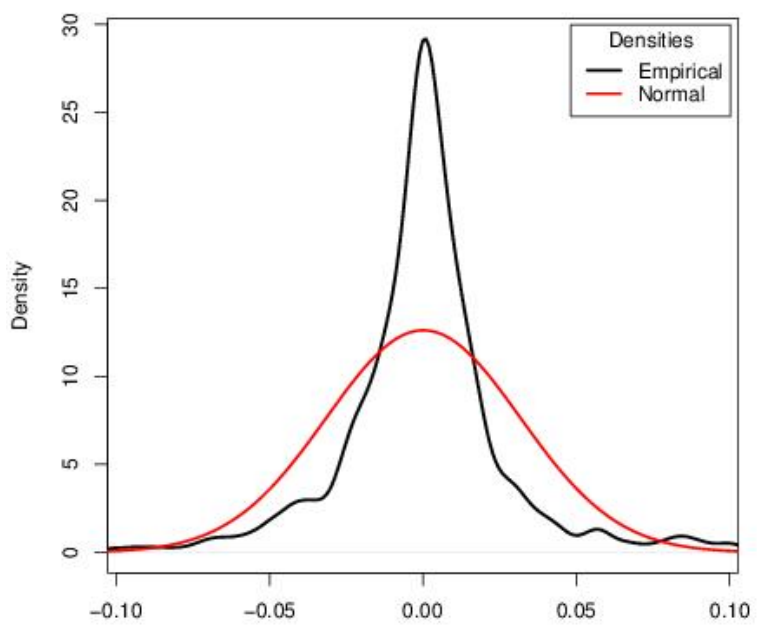

Kenya Commercial Bank (KCB). Daily log return January 2000 to August 2005

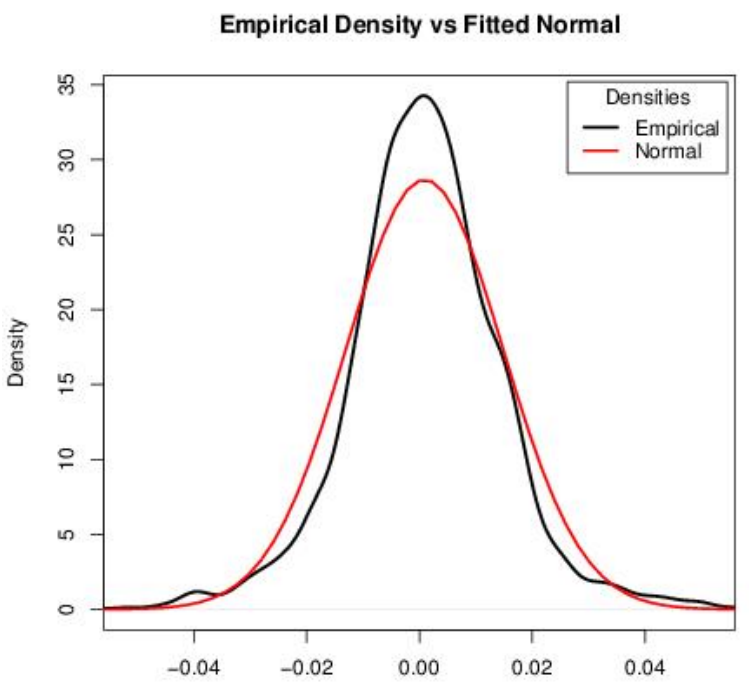

Royal Bank of Canada (RY). Daily log return January 2000 to August 2005

Figure 1. MLE of Normal distribution and Kernel densities for KCB and RY compared

Clearly the normal distribution does not reflect the empirical distribution. Similar results were obtained for the other six equities. This supports the fact that the simplifying assumptions of geometric 
Brownian motion about the dynamics of the underlying assets, on many occasions, is dotted with several shortcomings as pointed out in Schoutens [9]. Many modifications of modeling returns have been proposed and among them is the generalized hyperbolic distribution $(\mathrm{GH})$.

\section{The Generalized Hyperbolic Distribution}

The GH distribution possesses a lot of attractive properties, such as asymmetry, skewness and presence of semi-heavy tails. Therefore, the classes of GH distribution appears to be a good candidate for modeling $\log$ returns. In this section we provide a definition of the generalized hyperbolic distribution along with its classical representation as a variance mean-mixture of the normal with Generalized Inverse Gaussian(GIG) distribution. Hyperbolic and variance gamma distributions as special cases of GH are defined.

The random variable $W$ is said to have a generalized inverse gaussian (GIG) distribution if its probability density function is

$$
f_{G I G}(w ; \lambda, \gamma, \delta)=\frac{(\gamma / \delta)^{\lambda}}{2 K_{\lambda}(\gamma \delta)} w^{\lambda-1} \exp \left(-\frac{1}{2}\left(\frac{\delta^{2}}{w}+\gamma^{2} w\right)\right), w>0
$$

where $K_{\lambda}$ is a modified Bessel function of the third kind with the index $\lambda$.

$$
\begin{aligned}
K_{\lambda}(\omega) & =\frac{1}{2} \int_{0}^{\infty} \exp \left[-\frac{\omega}{2}\left(v^{-1}+v\right)\right] v^{\lambda-1} d v \\
& =\int_{0}^{\infty} \exp (-\omega \cosh t) \cosh (\lambda t) d t
\end{aligned}
$$

The parameters $\lambda \in \mathbb{R}, \gamma \geq 0, \delta \geq 0$ such that $\gamma \neq \delta$ if either of them takes the value zero.

The random variable $X$ is said to have a normal mean-variance distribution if

$$
X:=\mu+\beta W+\sigma \sqrt{W} Z
$$

where $Z \sim N(0,1), W$ is a positive random variable independent of $Z ; \mu$ and $\beta$ are real numbers and $\sigma$ is a positive real number. From the definition, we can see that the conditional distribution of $X$ given $W$ is normal with mean $\mu+\beta W$ and variance $\sigma^{2} W$.

Note that if the mixture variable $W$ is $\operatorname{GIG}(\lambda, \gamma, \delta)$ distributed, then $X$ is a generalized hyperbolic distribution with the $(\lambda, \alpha, \beta, \delta, \mu)$ parametrization, where $\alpha^{2}=\gamma^{2}+\beta^{2}$. The probability density function of the one-dimensional generalized hyperbolic distribution is given by the following equation,

$$
f_{G H}(x ; \alpha, \beta, \delta, \mu, \lambda)=\frac{(\gamma / \delta)^{\lambda}}{\sqrt{2 \pi} K_{\lambda}(\delta \gamma)} \cdot \frac{K_{\lambda-\frac{1}{2}}\left(\alpha \sqrt{\delta^{2}+(x-\mu)^{2}}\right)}{\left(\sqrt{\delta^{2}+(x-\mu)^{2}} / \alpha\right)^{\frac{1}{2}-\lambda}} \cdot e^{\beta(x-\mu)}
$$

In all cases, $\mu$ is the location parameter and can take any real value, $\delta$ is a scale parameter; where $\alpha$ and $\beta$ determine the distribution shape and $\lambda$ defines the subclasses of GH, and its related to the tail flatness as well. see Barndoff-Nielsen[8], McNeil et al [10] etc for more information.

\subsection{Hyperbolic Distributions and Variance Gamma Distribution}

Limiting distributions of the generalized hyperbolic distribution is obtained by varying parameter $\lambda$. When $\lambda=1$, and using the fact that $K_{1 / 2}(x)=\sqrt{\frac{\pi}{2}} x^{-1 / 2} e^{-x}$, we obtain the subclass of hyperbolic distribution with probability density function

$$
f_{\text {hyp }}(x ; \alpha, \beta, \delta, \mu)=\frac{\sqrt{\alpha^{2}-\beta^{2}}}{2 \alpha \delta K_{1}\left(\delta \sqrt{\alpha^{2}-\beta^{2}}\right)} \exp \left[-\alpha \sqrt{\delta^{2}+(x-\mu)^{2}}+\beta(x-\mu)\right] .
$$

When $\delta=0$, in equation (2), and using the fact that $K_{\lambda}(x) \sim \Gamma(\lambda) 2^{\lambda-1} x^{-\lambda}$, as $x \rightarrow 0$, we obtain

$$
f_{G H}(x ; \alpha, \beta, \mu, \lambda)=\frac{\left(\alpha^{2}-\beta^{2}\right)^{\lambda / 2}|x-\mu|^{\lambda-1 / 2}}{\sqrt{2 \pi} \alpha^{\lambda-1 / 2} 2^{\lambda-1}} e^{\beta(x-\mu)} K_{\lambda-1 / 2}(\alpha|x-\mu|) .
$$

If we take $\beta=\frac{\theta}{\sigma^{2}}, \lambda=1 / \nu$ and $\alpha=\sqrt{\beta^{2}+\left(2 \lambda / \sigma^{2}\right)}$, we obtain the probability density function of the variance gamma process (see Raible[11]) 


\subsection{Parameter Estimation}

Guided by the geometric Brownian motion assumption, that the daily log returns $x_{1}, x_{2}, \ldots, x_{n}$ are i.i.d.s, let the parameters to be estimated be denoted by $\Theta=(\lambda, \bar{\alpha}, \mu, \sigma, \gamma)$. We maximize

$$
\ln L_{X}\left(\Theta ; x_{1}, x_{2}, \ldots, x_{n}\right)=\sum_{i=1}^{n} \ln f_{X}\left(x_{i} ; \Theta\right) \text {. }
$$

The parameters of the mixture are found by introducing EM algorithm concept. It is assumed that log returns are the observed data and the incomplete data is generated by latent mixing variable $w_{1}, w_{2}, \ldots, w_{n}$ which is GIG distributed. Thus the joint log likelihood function

$$
\ln L_{X W}\left(\Theta ; x_{1}, \ldots, x_{n}, w_{1}, \ldots, w_{n}\right)=\sum_{i=1}^{n} \ln f_{X \mid W}\left(X_{i} \mid w_{i} ; \mu, \sigma, \gamma\right)+\sum_{i=1}^{n} \ln f_{W}\left(w_{i} ; \lambda, \bar{\alpha}\right)
$$

Calculate the conditional expectation E-step of the joint log-likelihood fuction given the data and the current estimates of the parameters followed by M-step Maximizing the objective function with respect to $\Theta$ to obtain an updated estimate. Repeating the E step and M step sequentially will obtain the maximum likelihood estimation of the parameter set $\Theta($ see $\mathrm{Hu}[12])$.

\section{Empirical Results}

\section{Parameter estimates of GH distribution and its subclasses}

Estimated parameters for the variance gamma distribution are given in Table 1. It is clear from both markets that the distributions are skewed. From Table 2, the absolute values of $\beta$ are greater than zero $(|\beta|>0)$ from both markets. One can observe that the underlying distribution is not only asymmetrical, but also leptokurtic. Note that for all the samples from NSE, the value of the scale parameter $\delta$ is very close to zero.

The variance gamma QQ-plots, GH QQ-plots, and their corresponding kernel densities are as shown in Figure 2. AL RY and KCB log returns fit VG well. The value of $\theta$ determines the nature of skewness for the variance gamma distribution. Most important, though we notice that both markets have different stochastic time. The value of $\nu$ in lies between $(0.52,0.87)$ which implies a higher frequency of business activity (time) compared to emerging market $(1.5,2.6)$ relatively lesser business activity in NSE in comparison to Montérial.

In most cases, the log-likelihood value for the fitted generalized hyperbolic distribution is higher than those of hyperbolic distribution and variance gamma. Table 3 gives the maximum likelihood estimates of generalized hyperbolic parameters. BBK KCB and RY fit the data well as evidenced by the QQ plots given in Figure 2.Therefore, the generalized hyperbolic distribution model, which is a five-parameter model, seems to fit the returns better, from both markets than models with fewer parameters like hyperbolic, variance gamma and gaussian distribution.

\section{Goodness of Fit Test and Frequency Distribution}

We analyze and compare the goodness of fit of the generalized hyperbolic distribution and some of their subclasses, using Kolmogorov distance and frequency distribution.

\section{Kolmogorov distance}

Kolmogorov distance is the supremum over the absolute differences between two density functions. Its expression is given by:

$$
K S=\sup _{x \in \mathbf{R}}\left|F_{e m p}(x)-F_{e s t}(x)\right|
$$


Table 1. MLE parameters of Variance gamma density fitted Montérial and NSE data

\begin{tabular}{lrrrrr}
\hline Co. & $\sigma$ & $\nu$ & $\theta$ & $\mu$ & LLF \\
\hline AEM & $3.0731 \mathrm{e}-02$ & 0.569706 & $4.0296 \mathrm{e}-03$ & $-3.6020 \mathrm{e}-03$ & 2960.673 \\
AL & $2.0313 \mathrm{e}-02$ & 0.532999 & $1.2492 \mathrm{e}-03$ & $-1.2469 \mathrm{e}-03$ & 3547.072 \\
RY & $1.3709 \mathrm{e}-02$ & 0.582838 & $7.0330 \mathrm{e}-04$ & $2.9993 \mathrm{e}-04$ & 4110.377 \\
SLF & $1.8965 \mathrm{e}-02$ & 0.873982 & $1.1115 \mathrm{e}-03$ & $-2.6059 \mathrm{e}-06$ & 3543.324 \\
\hline \hline BBK & $1.5454 \mathrm{e}-02$ & 1.543812 & $-4.1409 \mathrm{e}-04$ & $1.00995 \mathrm{e}-03$ & 4075.327 \\
KCB & $2.7678 \mathrm{e}-02$ & 2.150426 & $2.0188 \mathrm{e}-03$ & $4.11348 \mathrm{e}-10$ & 3227.641 \\
KQ & $2.6047 \mathrm{e}-02$ & 2.571561 & $6.9144 \mathrm{e}-04$ & $3.8438 \mathrm{e}-10$ & 3722.603 \\
EABL & $2.1660 \mathrm{e}-02$ & 1.705037 & $1.68104 \mathrm{e}-03$ & $1.01118 \mathrm{e}-03$ & 3542.802 \\
\hline
\end{tabular}

Table 2. MLE parameters of Hyperbolic distribution fitted for Montréal and NSE data

\begin{tabular}{lrrrrr}
\hline Co. & $\alpha$ & $\beta$ & $\delta$ & $\mu$ & LLF \\
\hline AEM & 53.4421 & 4.30726 & $1.837152 \mathrm{e}-02$ & $-3.634096 \mathrm{e}-03$ & 2963.292 \\
AL & 81.7215 & 3.00987 & $1.310892 \mathrm{e}-02$ & $-1.241524 \mathrm{e}-03$ & 3548.007 \\
RY & 118.0387 & 3.43599 & $7.72583 \mathrm{e}-03$ & $3.571136 \mathrm{e}-04$ & 4112.330 \\
SLF & 78.6708 & 3.53894 & $4.845935 \mathrm{e}-03$ & $-1.421884 \mathrm{e}-04$ & 3545.444 \\
\hline \hline BBK & 96.29101 & -1.628226 & $9.984908 \mathrm{e}-08$ & $9.5041 \mathrm{e}-08$ & 4054.704 \\
KCB & 52.47160 & 0.457439 & $4.733096 \mathrm{e}-07$ & $3.25479 \mathrm{e}-04$ & 3171.505 \\
KQ & 74.35194 & 2.753337 & $2.399309 \mathrm{e}-07$ & $5.68611 \mathrm{e}-4$ & 3688.820 \\
EABL & 69.75716 & 2.761331 & $3.247389 \mathrm{e}-07$ & $5.840876 \mathrm{e}-04$ & 3509.165 \\
\hline
\end{tabular}

where $F_{\text {emp }}$ and $F_{\text {est }}$ are the empirical and the estimated CDFs respectively. Kolmogorov distance is used because it pays more attention to the tails of distributions see Gyórfi et al. [13] and Prause [14]. The Kolmogorov distances of the normal, the hyperbolic, the variance gamma and the generalized hyperbolic distributions are presented in Table 4. From Table 4, we make inference based on the p-value being the measure of how much evidence one can have against the null hypothesis. The general rule is that a small p-value is evidence against the null hypothesis, while a large p-value means no evidence against the null hypothesis. At $1 \%$ level of significance, level we accept the null hypothesis that the data fits the three models for all the log returns from Montréal exchange. Generalized hyperbolic distribution appears to fit well the data in both markets. BBK and EABL of the Nairobi Securities Exchange fit VG at $1 \%$ level of confidence. Unfortunately at all levels, of all the four stocks from NSE considered, hyperbolic distribution is rejected .

\section{Frequency distributions}

In each column of the table, the relative frequencies of the returns in the intervals $(-k \hat{\sigma}, k \hat{\sigma})$ i.e $(P(|x|<$ $k \hat{\sigma}), k=1,2,3,4,5$. and $\hat{\sigma}$ is the standard deviation) are compared with the probabilities of the fitted distributions. We observe from Table 5 and Table 6 that among the fitted distributions, the probabilities of the generalized hyperbolic distribution are closer to the empirical (EMP) probabilities in most cases.

\section{Concluding Remarks}

In this study, we have tried to investigate geometric Browinan assumption of modeling univariate underlying process from an empirical perspective. We fitted four asset prices from the Montréal exchange and four asset prices from Nairobi Securities Exchange to models based on normality assumption and generalized hyperbolic distribution and its subclasses. Empirical evidence suggest that the underlying distribution of log returns is heavy-tailed in both markets and not normally distributed as provided for by the geometric Brownian motion assumption. About $83-84 \%$ of empirical data from the Nairobi Securities 
Table 3. Generalized hyperbolic parameter estimates for Montréal and NSE data

\begin{tabular}{lrrrrrr}
\hline Co. & $\lambda$ & $\alpha$ & $\beta$ & $\delta$ & $\mu$ & LLF \\
\hline AEM & $-1.43421 \mathrm{e}-00$ & 22.45251 & 3.182777 & 0.04385096 & $-3.10649 \mathrm{e}-03$ & 2968.365 \\
AL & $-4.47653 \mathrm{e}-02$ & 66.30114 & 3.247621 & 0.0211068 & $-1.34398 \mathrm{e}-03$ & 3548.421 \\
RY & $-3.90192 \mathrm{e}-01$ & 90.85274 & 3.480525 & 0.01536024 & $4.77593 \mathrm{e}-04$ & 4113.503 \\
SLF & $-9.69996 \mathrm{e}-01$ & 24.72129 & 1.517417 & 0.01800045 & $3.79528 \mathrm{e}-04$ & 3555.739 \\
\hline BBK & $-5.81346 \mathrm{e}-01$ & 20.63537 & -1.00749 & $7.77159 \mathrm{e}-03$ & $9.05012 \mathrm{e}-04$ & 4109.781 \\
KCB & $3.75006 \mathrm{e}-02$ & 22.39679 & $9.50887 \mathrm{e}-02$ & $5.91669 \mathrm{e}-03$ & $5.78596 \mathrm{e}-04$ & 3222.247 \\
KQ & $-4.91616 \mathrm{e}-01$ & 6.32854 & 1.66513 & $7.37481 \mathrm{e}-03$ & $1.01943 \mathrm{e}-03$ & 3764.946 \\
EABL & $-1.75863 \mathrm{e}-01$ & 23.86991 & $4.13654 \mathrm{e}-01$ & $6.52984 \mathrm{e}-03$ & $1.01915 \mathrm{e}-03$ & 3559.446 \\
\hline
\end{tabular}

Table 4. Kolmogorov distances

\begin{tabular}{ll|l||l|l||l|l||l|l}
\hline & \multicolumn{2}{l|}{ Normal } & \multicolumn{2}{l|}{ Generalized H } & \multicolumn{2}{l|}{ VGamma } & \multicolumn{2}{l}{ Hyperbolic } \\
\cline { 2 - 8 } & K $_{\text {Dist }}$ & p-value & K $_{\text {Dist }}$ & p-value & K $_{\text {Dist }}$ & p-value & K$_{\text {Dist }}$ & p-value \\
\hline \hline AEM & 0.0706 & $1.483 \mathrm{e}-06$ & 0.0423 & $1.243 \mathrm{e}-02$ & 0.0311 & $1.301 \mathrm{e}-01$ & 0.0289 & $1.863 \mathrm{e}-01$ \\
AL & 0.0459 & $5.143 \mathrm{e}-03$ & 0.0261 & $2.888 \mathrm{e}-01$ & 0.0282 & $2.088 \mathrm{e}-01$ & 0.0233 & $4.258 \mathrm{e}-01$ \\
RY & 0.0543 & $4.641 \mathrm{e}-04$ & 0.0212 & $5.491 \mathrm{e}-01$ & 0.0219 & $5.064 \mathrm{e}-01$ & 0.0191 & $6.823 \mathrm{e}-01$ \\
SLF & 0.0845 & $7.252 \mathrm{e}-09$ & 0.0309 & $1.496 \mathrm{e}-01$ & 0.0265 & $2.968 \mathrm{e}-01$ & 0.0198 & $6.558 \mathrm{e}-01$ \\
\hline BBK & 0.1318 & $2.2 \mathrm{e}-16$ & 0.0298 & $1.640 \mathrm{e}-01$ & 0.0333 & $8.733 \mathrm{e}-02$ & 0.0376 & $3.373 \mathrm{e}-02$ \\
KCB & 0.1387 & $2.2 \mathrm{e}-16$ & 0.0407 & $1.922 \mathrm{e}-02$ & 0.0536 & $6.437 \mathrm{e}-04$ & 0.0508 & $1.438 \mathrm{e}-03$ \\
KQ & 0.1595 & $2.2 \mathrm{e}-16$ & 0.0262 & $2.864 \mathrm{e}-01$ & 0.0532 & $6.892 \mathrm{e}-04$ & 0.0631 & $2.661 \mathrm{e}-05$ \\
EABL & 0.1577 & $2.2 \mathrm{e}-16$ & 0.0247 & $3.708 \mathrm{e}-01$ & 0.0298 & $1.736 \mathrm{e}-01$ & 0.0509 & $1.614 \mathrm{e}-03$ \\
\hline
\end{tabular}

Exchange lies between the first standard deviation compared to 72-75\% from Montérial Exchange instead of $68 \%$ if log returns were to be normally distributed. This implies that the normality assumption may not be the best option for modeling returns.

It is clear from our study that emerging market and developed market too are affected by shocks that produce a diversity of jumps. One can argue, from the estimated Kolmogorov distance in addition to calculated p-values and QQ plots, that normal distribution which is a two-parameter function, gives poor fit. Variance gamma fit the log returns of developed market and only two of the companies from emerging market. The hyperbolic distribution seems inferior when modeling returns from emerging market as opposed to developed market. However Generalized hyperbolic distribution, which is a five-parameter density seems to explain better the behavior of the empirical distribution of log returns from both markets. This seems to confirm more about stylist facts observed in existing literature, in any market. The data used was not large enough to make a generalized observation. Empirically, both markets are not operating at the same business time and the log return are not normally distributed. It would be interesting to incorporate serial joint dependency in the future studies.

Acknowledgement. Useful suggestions and valuable comments by the anonymous reviewer are highly acknowledged.

\section{References}

1. F. Black and M. Scholes, "The pricing of Options and Corporate liabilities," Journal of Political Economy, vol. 81, pp. 659-683, 1973.

2. R. Merton, "Theory of rational option pricing," Bell J. Economics and Management Science, vol. 4, pp. 141-183, 1973.

3. R. Cont, "Empirical properties of asset returns: stylized facts and statistical issues," Quantitative Finance, vol. 1, pp. 223-226, 2001. 
4. D. Madan and E. Seneta, "The variance gamma(V.G.) model for share markets," Journal of Bussiness, vol. 63, pp. 511-524, 1990.

5. D. Madan, C. Chang, and P. Carr, "The variance gamma process and option pricing," European Finance Review, vol. 2, pp. 79-105, 1998.

6. P. Carr and L. Wu, "Time-Changed Lévy processes and option pricing," Journal of Financial Economics, vol. 71, pp. 113-141, 2004.

7. E. Eberlein and U. Keller, "Hyperbolic Distributions in Finance," Bernaoulli, vol. 1, pp. 281-299, 1995.

8. O. Barndorff-Nielsen, "Exponentially decreasing distributions for logarithm of particle size," Proceedings Royal Society London Ser A, vol. 353, pp. 401-419, 1977.

9. W. Schoutens, Lévy Processes in Finance Pricing Financial Derivatives, 1st ed. Wiley Publishers, 2003.

10. A. McNeil, R. Frey, and P. Embrechts, Quantitative Risk management: Concepts Techniques and Tools . Priceton University Press, 2005.

11. S. Raible, "Lévy processes in Finance: Theory, Numerics, and Empirical Facts," Ph.D. dissertation, University of Freiburg., 2000.

12. W. Hu, "Calibration of Multivariate Generalized Hyperbolic Distributions Using the EM Algorithm, with applications in Risk management, Portfolio Optimization and Portfolio Credit Risk." Ph.D. dissertation, The Florida University, 2005.

13. L. Gyórfi, I. Vajda, and E. Meulen, "Minimum Kolmogorov Distance Estimates of Parameters and Parametrizated Distributions," Metrica, vol. 43, pp. 237-255, 1996.

14. K. Prause, "The Generalized Hyperbolic Model: Estimation,Finsncial Derivatives and Risk Measures," Ph.D. dissertation, University of Freiburg, 1999.

\section{List of Abbreviations}

The following set of notations were used throughout the article. They range from distributions and names of the listed companies in Nairobi securities exchange and Montréal exchange respectively.

GH-generalised hyperbolic distribution

HY-hyperbolic distribution

NM- Normal distribution

VG-Variance gamma distribution

GIG-generalized inverse Gaussian distribution

EMP-Empirical density function

KS-Kolmogorov distance

QQ-Quantile-Quantile plots

NSE- Nairobi Securities Exchange

BBK-Barclays Bank of Kenya

KCB-Kenya Commercial Bank

EABL-East African Breweries Limited

KQ- Kenya Airways

Mx - Montréal Exchange

AEM-Angnico Eagle Mines limited

AL- Alcan Inc

RY-Royal Bank of Canada

SLF-Sun life financial 
KCB Itd Variance Gamma qqlot

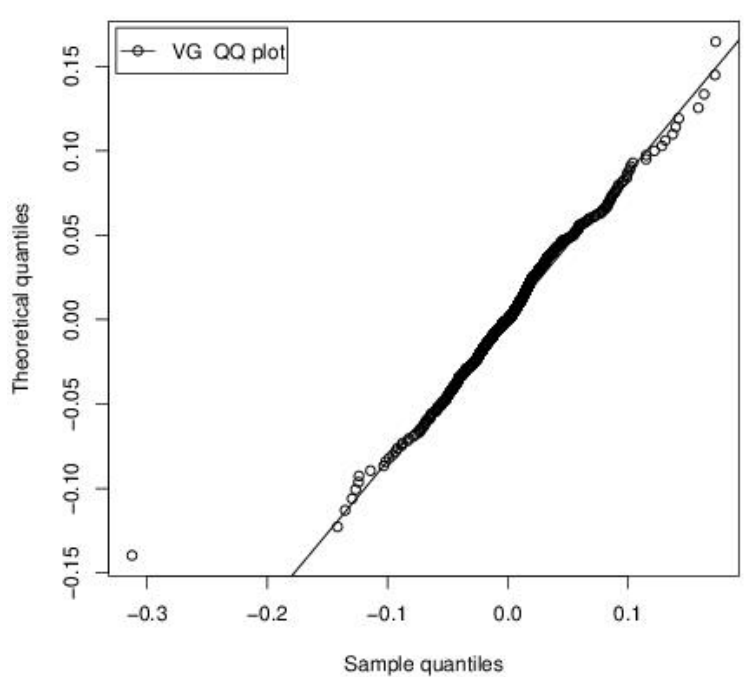

Barclays Bank Kenya GH qqlot

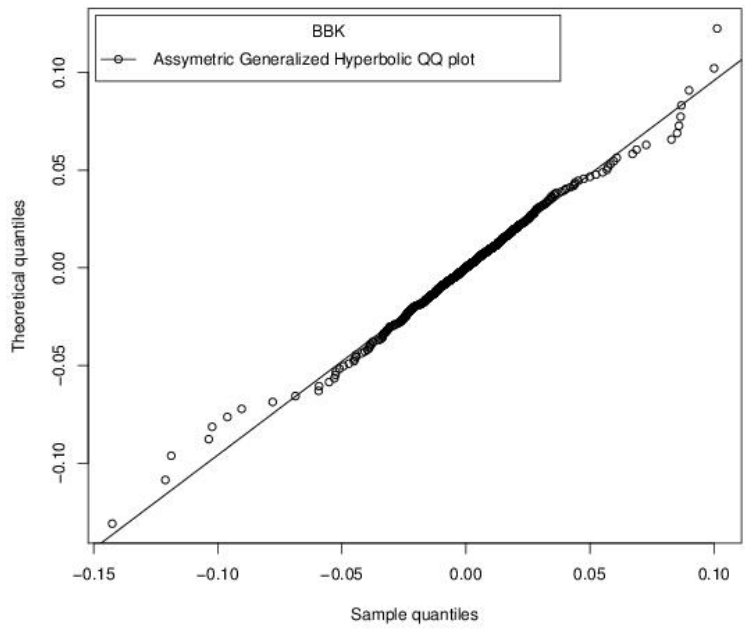

Empirical Density vs Fitted Normal,GH,HY,VG densities

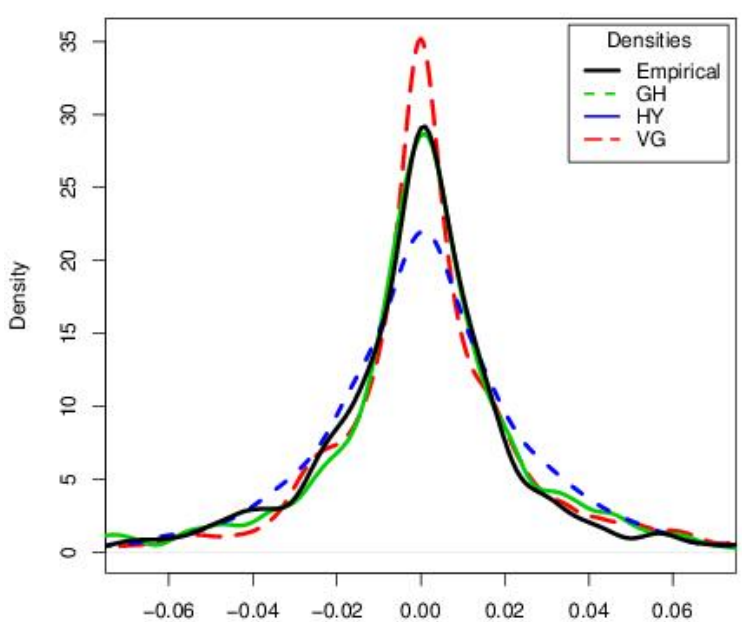

Kenya Commercial Bank (KCB) daily log returns from January 2000 to August 20
RY Itd Variance Gamma qqlot

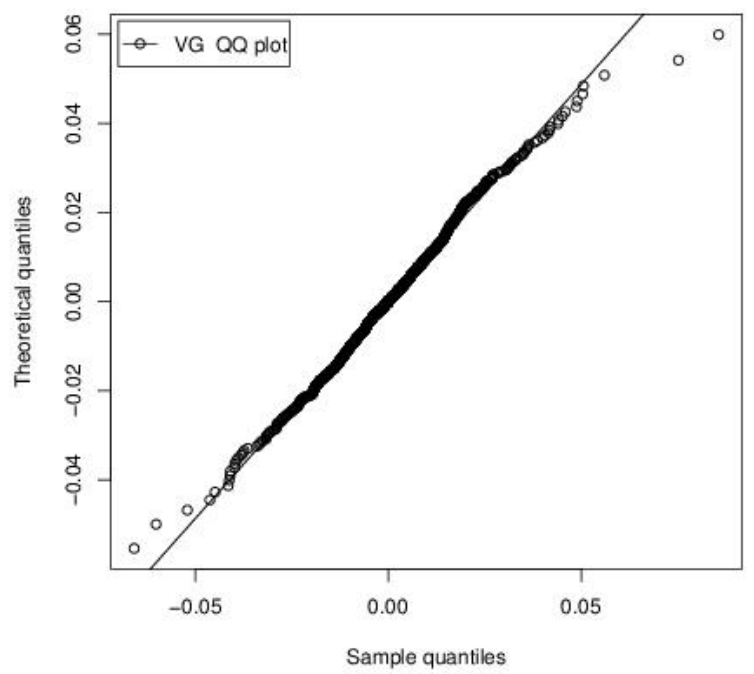

AL Asymetric Generaized Hyperbolic QQ plot

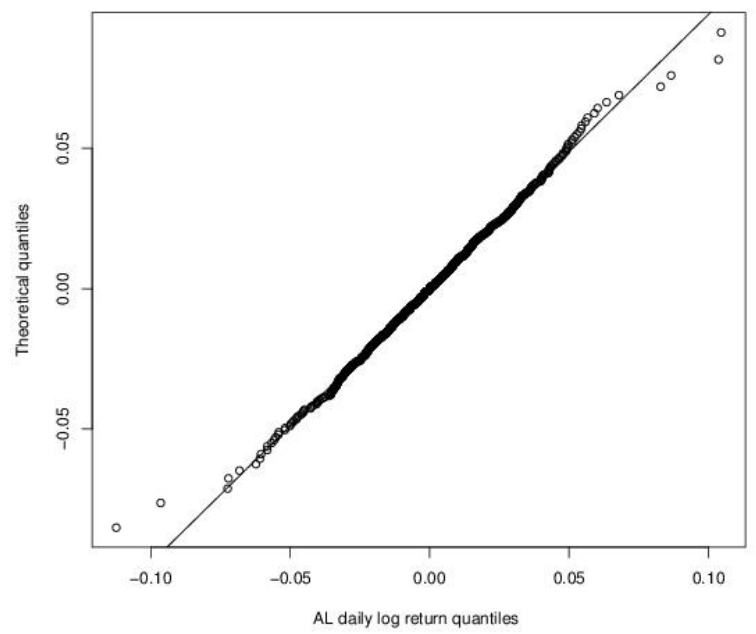

Empirical Density vs Fitted Normal,GH,HY,VG densities

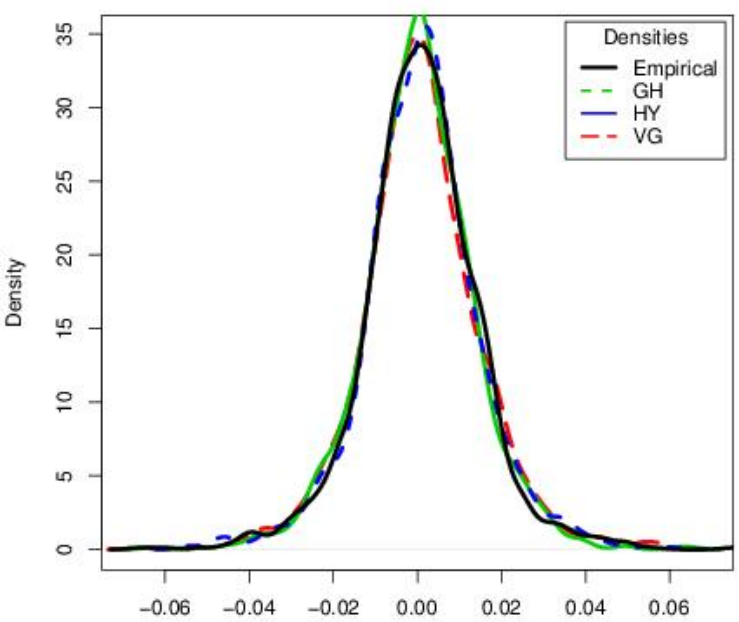

Royal Bank of Cananda (RY) daily log returns from January 2000 to August 2005 
Table 5. Frequency distribution of log returns from Montérial Exchange

\begin{tabular}{llllllll}
\hline \hline Co. & Density & $|x|<\hat{\sigma}$ & $<2 \hat{\sigma}$ & $<3 \hat{\sigma}$ & $<4 \hat{\sigma}$ & $<5 \hat{\sigma}$ & $|x|>5 \hat{\sigma}$ \\
\hline AEM & EMP & 0.7558 & 0.9569 & 0.9894 & 0.9950 & 0.9971 & $1.07268 \mathrm{e}-01$ \\
& NM & 0.6826 & 0.9544 & 0.9973 & 0.9999 & 0.9999 & $5.7334 \mathrm{e}-07$ \\
& HY & 0.7493 & 0.9499 & 0.9902 & 0.9981 & 0.9996 & $3.7232 \mathrm{e}-04$ \\
& VG & 0.7465 & 0.9497 & 0.9907 & 0.9983 & 0.9997 & $2.8439 \mathrm{e}-04$ \\
& GH & 0.7560 & 0.9468 & 0.9869 & 0.9962 & 0.9988 & $1.1885 \mathrm{e}-03$ \\
\hline AL & EMP & 0.7374 & 0.9484 & 0.9915 & 0.9957 & 0.9978 & $3.0345 \mathrm{e}-02$ \\
& NM & 0.6826 & 0.9544 & 0.9972 & 0.9999 & 0.9999 & $5.7406 \mathrm{e}-07$ \\
& HY & 0.7376 & 0.9462 & 0.9894 & 0.9979 & 0.9996 & $3.9088 \mathrm{e}-04$ \\
& VG & 0.7348 & 0.9461 & 0.9901 & 0.9982 & 0.9997 & $2.8777 \mathrm{e}-04$ \\
& GH & 0.7418 & 0.9479 & 0.9893 & 0.9977 & 0.9996 & $4.9973 \mathrm{e}-04$ \\
\hline RY & EMP & 0.7459 & 0.9470 & 0.9872 & 0.9964 & 0.9985 & $7.0571 \mathrm{e}-03$ \\
& NM & 0.6816 & 0.9540 & 0.9972 & 0.9999 & 0.9999 & $6.0508 \mathrm{e}-07$ \\
& HY & 0.7426 & 0.9464 & 0.9892 & 0.9978 & 0.9995 & $4.2351 \mathrm{e}-04$ \\
& VG & 0.7395 & 0.9461 & 0.9897 & 0.9981 & 0.9996 & $3.2644 \mathrm{e}-04$ \\
& GH & 0.7518 & 0.9516 & 0.9901 & 0.9978 & 0.9995 & $4.7676 \mathrm{e}-04$ \\
\hline SLF & EMP & 0.7972 & 0.9456 & 0.9779 & 0.9955 & 0.9977 & $3.6737 \mathrm{e}-02$ \\
& NM & 0.6821 & 0.9542 & 0.9972 & 0.9999 & 0.9999 & $5.8953 \mathrm{e}-07$ \\
& HY & 0.7647 & 0.9487 & 0.9888 & 0.9975 & 0.9994 & $5.3066 \mathrm{e}-04$ \\
& VG & 0.7627 & 0.9469 & 0.9883 & 0.9974 & 0.9994 & $5.5580 \mathrm{e}-04$ \\
& GH & 0.7793 & 0.9447 & 0.9820 & 0.9931 & 0.9971 & $2.8843 \mathrm{e}-03$ \\
\hline \hline
\end{tabular}

Table 6. Frequency distribution of log returns from Nairobi Securities Exchange

\begin{tabular}{llllllll}
\hline \hline Co. & Density & $|x|<\sigma$ & $<2 \sigma$ & $<3 \sigma$ & $<4 \sigma$ & $<5 \sigma$ & $|x|>5 \sigma$ \\
\hline BBK & EMP & 0.8454 & 0.9553 & 0.9787 & 0.9879 & 0.9929 & $2.5513 \mathrm{e}-02$ \\
& NM & 0.6825 & 0.9544 & 0.9972 & 0.9999 & 0.9999 & $5.7815 \mathrm{e}-07$ \\
& HY & 0.8153 & 0.9660 & 0.9937 & 0.9988 & 0.9997 & $2.1305 \mathrm{e}-04$ \\
& VG & 0.8141 & 0.9565 & 0.9892 & 0.9972 & 0.9993 & $6.9737 \mathrm{e}-04$ \\
& GH & 0.8410 & 0.9501 & 0.9790 & 0.9899 & 0.9947 & $5.2044 \mathrm{e}-03$ \\
\hline EABL & EMP & 0.8364 & 0.9534 & 0.9811 & 0.9920 & 0.9934 & $5.0145 \mathrm{e}-02$ \\
& NM & 0.6818 & 0.9541 & 0.9972 & 0.9999 & 0.9999 & $5.9781 \mathrm{e}-07$ \\
& HY & 0.8204 & 0.9676 & 0.9941 & 0.9989 & 0.9998 & $1.9446 \mathrm{e}-04$ \\
& VG & 0.8169 & 0.9551 & 0.9882 & 0.9967 & 0.9991 & $8.9893 \mathrm{e}-04$ \\
& GH & 0.8324 & 0.9465 & 0.9792 & 0.9912 & 0.9960 & $3.9322 \mathrm{e}-03$ \\
\hline KCB & EMP & 0.8255 & 0.9406 & 0.9771 & 0.9899 & 0.9964 & $9.5067 \mathrm{e}-02$ \\
& NM & 0.6826 & 0.9544 & 0.9972 & 0.9999 & 0.9999 & $5.7349 \mathrm{e}-07$ \\
& HY & 0.8094 & 0.9636 & 0.9930 & 0.9986 & 0.9997 & $2.5145 \mathrm{e}-04$ \\
& VG & 0.8272 & 0.9543 & 0.9868 & 0.9960 & 0.9987 & $1.2368 \mathrm{e}-03$ \\
& GH & 0.8191 & 0.9424 & 0.9787 & 0.9915 & 0.9965 & $3.4884 \mathrm{e}-03$ \\
\hline KQ & EMP & 0.8454 & 0.9376 & 0.9666 & 0.9900 & 0.9971 & $5.8114 \mathrm{e}-02$ \\
& NM & 0.6819 & 0.9541 & 0.9972 & 0.9999 & 0.9999 & $5.9651 \mathrm{e}-07$ \\
& HY & 0.8201 & 0.9675 & 0.9941 & 0.9989 & 0.9998 & $1.9516 \mathrm{e}-04$ \\
& VG & 0.7759 & 0.9208 & 0.9695 & 0.9878 & 0.9949 & $5.0005 \mathrm{e}-03$ \\
& GH & 0.8298 & 0.9270 & 0.9588 & 0.9739 & 0.9822 & $1.7734 \mathrm{e}-02$ \\
\hline \hline
\end{tabular}

